\title{
Francisco José Zamith Guimarães
}

\section{Utilização de ontologias no domínio B2C}

\author{
Dissertação de Mestrado
}

DEPARTAMENTO DE INFORMÁTICA

Programa de Pós-Graduação em Informática 


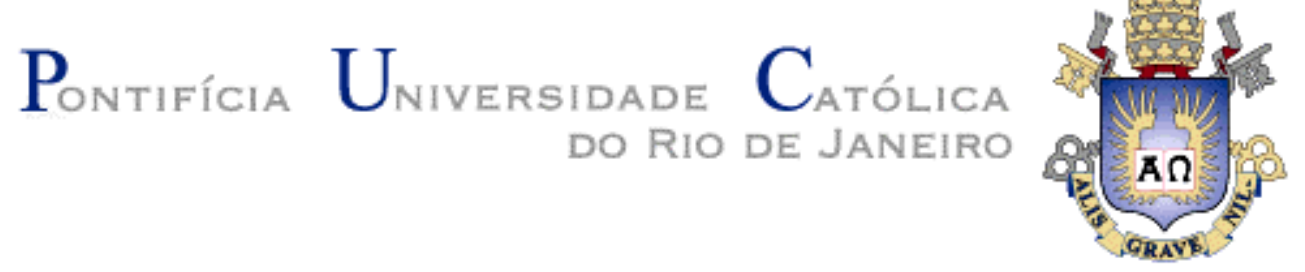

Francisco José Zamith Guimarães

Utilização de ontologias no domínio B2C

Dissertação de Mestrado

Dissertação apresentada como requisito parcial para obtenção do título de Mestre pelo Programa de Pós-Graduação em Informática da PUC-Rio.

Orientador: Prof. Carlos José Pereira de Lucena

Rio de Janeiro, novembro de 2002 


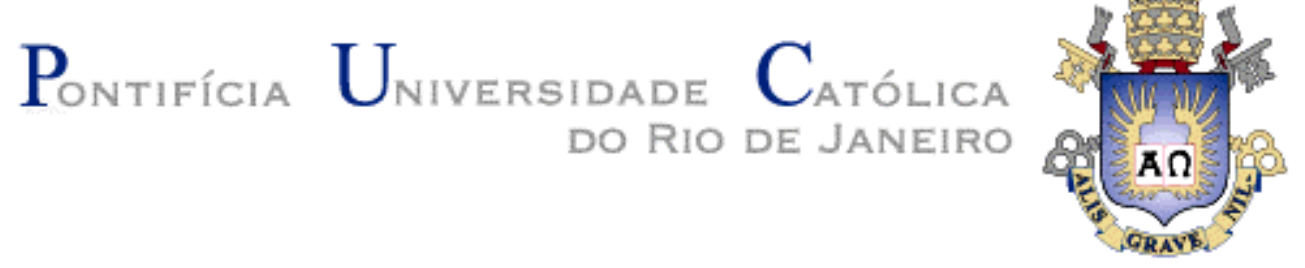

Francisco José Zamith Guimarães

Utilização de ontologias no domínio B2C

Dissertação apresentada como requisito parcial para obtenção do título de Mestre pelo Programa de PósGraduação em Informática da PUC-Rio. Aprovada pela Comissão Examinadora abaixo assinada.

Prof. Carlos José Pereira de Lucena Orientador PUC-Rio

Prof. Arndt von Staa PUC-Rio

Prof. Hugo Fuks PUC-Rio

Profa. Simone Diniz Junqueira Barbosa PUC-Rio

Prof. Ney Dumont Coordenador(a) Setorial do Centro Técnico Científico - PUC-Rio 
Todos os direitos reservados. É proibida a reprodução total ou parcial do trabalho sem autorização da universidade, do autor e do orientador.

\section{Francisco José Zamith Guimarães}

Graduou-se em Engenharia da Computação na Pontifícia Universi-dade Católica do Rio de Janeiro em 2000. Desenvolve pesquisas sobre ontologias e Web semântica.

Ficha Catalográfica

Guimarães, Francisco José Zamith

Utilização de ontologias no domínio B2C / Francisco José Zamith Guimarães ; orientador: Carlos José Pereira de Lucena. - Rio de Janeiro : PUC, Departamento de Informática, 2002.

[14], 195 f. : il. ; $30 \mathrm{~cm}$

Dissertação (mestrado) - Pontifícia Universidade Católica do Rio de Janeiro, Departamento de Informática. Inclui referências bibliográficas.

Incluí referências bibliográficas.

1. Informática - Teses. 2. Ontologias. 3. B2C. 4. Web semântica. I. Lucena, Carlos José Pereira de. II. Pontifícia Universidade Católica do Rio de Janeiro. Departamento de Informática. III. Título. 
Para meus pais, Martha e Francisco Pelo apoio e incentivo. 


\section{Agradecimentos}

Aos meus pais, pelo apoio e incentivo.

Aos meus avós, pelo carinho.

A minha irmã, pelo companheirismo.

A Danielle, pelo amor e paciência.

Ao meu orientador Professor Carlos José Pereira de Lucena pelo apoio e auxílio para a realização deste trabalho.

Ao CNPq e à PUC-Rio, pelos auxílios concedidos, sem os quais este trabalho não poderia ter sido realizado.

Aos meus amigos da PUC-Rio.

Aos professores que participaram da Comissão examinadora. 
A todas as pessoas do Departamento de Informática.

A todos os meus familiares e amigos que de uma forma ou de outra me estimularam ou me ajudaram. 


\section{Resumo}

Guimarães, Francisco José Zamith; Lucena, Carlos José Pereira. Utilização de ontologias no domínio B2C. Rio de Janeiro, 2002. 195p. Dissertação de Mestrado - Departamento de Informática, Pontifícia Universidade Católica do Rio de Janeiro.

A principal dificuldade dentro do domínio B2C está em aumentar a utilidade da WWW para o comércio eletrônico através da melhoria das possibilidades oferecidas ao consumidor. Apesar de a WWW permitir ao comprador ter acesso a uma grande quantidade de informação, obter a informação do fornecedor certo que venda o produto desejado a um preço razoável, pode ser uma tarefa muito custosa. Uma das formas de melhorar essa situação é através do uso de agentes inteligentes de busca de informação, isto é, agentes de compra, que auxiliam os compradores a encontrar produtos de seu interesse. Para que isso ocorra esbarra-se em uma dificuldade inerente à própria WWW: a mistura da linguagem natural, imagens e informação de layout de HTML são uma das maiores barreiras para a automatização do comércio eletrônico, pois a semântica da informação é somente compreensível por seres humanos. Desta forma espera-se conseguir agentes de compra mais eficientes quando associados ao uso de ontologias, e lojas virtuais que tenham anotações especiais que sigam uma ontologia. Nessa dissertação fazemos um estudo sobre as principais tecnologias envolvidas no desenvolvimento de ontologias em Ciência da Computação. Fazemos também um estudo de caso sobre a aplicação de ontologias dentro do domínio de B2C, visando assim avaliar o potencial $\mathrm{e}$ as dificuldades existentes para $\mathrm{o}$ desenvolvimento desse tipo de aplicação.

\section{Palavras-chave}

Informática; Ontologias; B2C; Web Semântica. 


\section{Abstract}

Guimarães, Francisco José Zamith; Lucena, Carlos José Pereira (Advisor). Ontologies use in B2C domain. Rio de Janeiro, 2002. 195p. MSc. Dissertation - Departamento de Informática, Pontifícia Universidade Católica do Rio de Janeiro.

The main difficulty associated with the B2C domain is increasing the usefulness of WWW for the electronic trade through the improvement of the services provided to the consumer. Even though the WWW allows the buyer to have access to a great amount of information, to obtain the information from the right supplier that sells the desired product by a reasonable price can be a very expensive task. One of the ways of improving the web functionality is through the use of intelligent agents for search of information, that is, the introduction of purchase agents that aid the buyers to find products of their interest. For that to happen we need to overcome an inherent difficulty of the WWW: the mixture of natural language, images and layout information in HTML is one of the greatest barriers for the automation of the electronic trade, because the semantics of the information is only comprehensible for human beings. To solve this problem we hope to produce more efficient purchase agents by associating them to the use of ontologies, and virtual stores that have special annotations that follow ontologies. In the present dissertation we make a study of the main technologies related to ontologies development in computer science. We also develop a case study about the ontologies application to the $\mathrm{B} 2 \mathrm{C}$ domain, seeking in this way to evaluate potential and existing difficulties for the development of this type of application.

\section{Keywords}

Computer science; Ontology; B2C; Web Semantics. 


\section{Sumário}

1 Introdução

2 Business to Consumer 23

2.1. Situação atual 23

2.2. Comportamento do consumidor 27

2.3. Relacionamento Comprador - Vendedor 32

3 Ferramentas de busca 34

3.1. Máquina de busca genérica 35

3.1.1. Percorrendo a Internet 35

3.1.2. Mantendo um índice 37

3.1.3. Construindo a busca 37

3.2. Meta máquina de busca $\quad 40$

3.3. Softbots 43

3.3.1. Shopbots 43

3.3.2. Wrappers e a Web 46

4 Ontologia 49

4.1. O que é ontologia 49

4.2. Vantagens do uso de ontologias 53

4.3. O que compõe uma ontologia 55

4.4. Classificação das ontologias 56

4.5. Principais áreas de aplicação das ontologias 58

4.5.1. Processamento de linguagem natural 58

4.5.2. Gestão do conhecimento 58

4.5.3. Web semântica $\quad 59$

4.5.4. Comércio eletrônico 59

4.6. Abordagens para o desenvolvimento de uma ontologia 61

4.6.1. Abordagem partindo de uma inspiração 61 
4.6.2. Abordagem indutiva 61

4.6.3. Abordagem dedutiva 62

4.6.4. Abordagem sintética 62

4.6.5. Abordagem colaborativa 63

4.7. Metodologias para o desenvolvimento de uma ontologia 64

4.7.1. Metodologia proposta por Mike Uschold e Martin King (Uschold \&

King, 1995) 65

4.7.2. Metodologia proposta por Michael Grüninger e Mark S. Fox

(Grüninger \& Fox, 1995) 65

4.7.3. Metodologia proposta por Mariano Fernández, Asunción Gómes-

Pérez e Natalia Juristo (METHONTOLOGY) (Fernández et al., 1997) 67

4.7.4. Conclusão a respeito das metodologias $\quad 72$

5 Tecnologias estudadas $\quad 73$

5.1. XML 74

5.1.1. Visão geral de um documento XML (Martin et al., 2001; Holzner, 2001; Bray et al., 1998) 75

5.1.2. Partes que formam um documento XML 78

5.1.3. Tecnologias auxiliares a XML 80

5.1.3.1. DTD e XML Schema 80

5.1.3.2. Namespace 82

5.2. RDF (Martin et al., 2001; Holzner, 2001; Swick \& Lassila, 1999) 85

5.2.1. Modelo básico 85

5.2.2. Sintaxe básica 88

5.2.2.1. Sintaxe serializada básica 88

5.2.2.2. Sintaxe abreviada básica 90

5.2.3. Coleções 92

5.2.4. Declaração sobre declaração RDF 95

5.3. RDF Schema 97

5.3.1. Vocabulário RDF Schema 98

5.4. DAML+OIL 103

5.4.1. Documento DAML+OIL 104

5.4.1.1. Cabeçalho do documento DAML+OIL 105 
5.4.1.2. Elemento de classe

5.4.1.3. Restrições de propriedade 107

5.4.1.4. Elemento de propriedade 110

5.4.1.5. Instâncias 112

5.5. SHOE 113

5.5.1. Vocabulário SHOE 113

5.6. Comparação entre as linguagens 115

6 Estudo de caso 119

6.1. Ontologia proposta para a aplicação 120

6.1.1. 2BuyNet (Fortunato, 1999) 122

6.2. Aplicação 124

6.2.1. Máquina de inferência $\quad 125$

6.2.2. Vantagens obtidas com o uso de ontologias 127

6.2.2.1. A descrição dos produtos de forma estruturada, e a separação das tags de layout da informação útil 128

6.2.2.2. Padronização da descrição de uma família de produtos 131

6.2.2.3. A capacidade de fazer inferências sobre as informações que seguem a ontologia 133

6.2.3. Dificuldades encontradas 134

6.2.3.1. Desempenho da aplicação 135

Expressividade da máquina de inferência 136

7 Conclusão 138

7.1. Principais contribuições 138

7.2. Trabalhos futuros 140

8 Referências Bibliograficas 141

$\begin{array}{ll}\text { Apêndice A - Telas do sistema } & 147\end{array}$

Apêndice B - Documentação ontologia 156

Documento sobre o objetivo da ontologia com breve descrição do domínio 
Árvores de classificação de conceitos

Diagramas de relações binárias 159

Dicionário de conceitos 160

Tabela de relações binárias 163

Tabela de atributos de instância 164

Tabela de axiomas 167

Tabela de instâncias 169

Apêndice C - Diagramas de classe da aplicação 171

Pacote responsável pela interface com o usuário (Interface) 172

Pacote responsável pela interface com a base de conhecimento

(BaseConhecimento) 177

Pacote responsável por controlar as classes das ontologias

(OntologiaControle)

Pacote responsável por formular as perguntas às ontologias(Pergunta)181

Pacote responsável pela fábrica de uma família de produtos (Fabricas)185

Apêndice D - Diagramas de seqüência da aplicação 


\section{Lista de Figuras}

Figura 1 - Estimativas de vários órgãos de pesquisa sobre o volume de transações B2C a serem realizadas nos Estados Unidos(OECD, 2001b).24 Figura 2 - Estimativas de vários órgãos de pesquisa sobre o volume de transações B2C a serem realizadas na Europa (OECD, 2001b).

Figura 3 - Volume de transações B2C na América Latina (Wenrich \& Becerra, 2001).

Figura 4 - Colocação das vendas, em valores, no segmento B2C por várias categorias no ano de 2000 , desconsiderando-se a categoria de leilões virtuais (OECD, 2002).

Figura 5 - Colocação das vendas no segmento de B2C na América Latina por várias categorias no ano de 2001 (Wenrich \& Becerra, 2001). 26

Figura 6 - Relação entre Indivíduos que usam a Internet versus Indivíduos que compra pela Internet em diversos países (OECD, 2001b). 26

Figura 7 - Processo de decisão de consumidor. 29

Figura 8 - Máquina de busca genérica. 39

Figura 9 - Meta máquina de busca genérica. 41

Figura 10 - Agente de compra (Shopbot) passivo de livros. 45

Figura 11 - Níveis de organização da informação na Web. 46

Figura 12 - Comparação entre ontologia e base de conhecimento. $\quad 52$

Figura 13 - Diferentes tipos de ontologias e seus relacionamentos. $\quad 57$

Figura 14 - Procedimento para o desenvolvimento e avaliação de ontologia.

Figura 15 - Visão geral do processo de desenvolvimento de ontologias (Fernández et al., 1997).

Figura 16 - Artefatos gerados na metodologia proposta em (Fernández et al., 1997; López et al., 1999).

Figura 17 - Organização em camada das linguagens.

Figura 18 - Representação gráfica de uma declaração RDF. $\quad 87$

Figura 19 -Declaração RDF com relacionamento entre recursos. 87 
Figura 20 - Declaração RDF que utiliza coleções.

Figura 21 - Declaração sobre declaração RDF. 96

Figura 22 - Funcionamento do 2BuyNet. 123

Figura 23 - Arquitetura da aplicação. 125

Figura 24 - Exemplo de inferência. 127

Figura 25 - Código 17 apresentado pelo Internet Explorer. $\quad 129$

Figura 26 - Mapeamento entre ontologias. 132

Figura 27 - Relacionamento entre livro, CD e produto. 134

Figura 28 - Aplicação com arquitetura distribuída. 136

Figura 29 - Tela inicial do sistema. 149

Figura 30 - Formulário do perfil da pessoa a ser presenteada. $\quad 149$

Figura 31 - Livros indicados ao perfil de pessoa. 150

Figura 32 - Formulário para busca de livros. 151

Figura 33 - Resposta do sistema a uma busca de livros. 151

Figura 34 - Detalhes de um Livro. 152

Figura 35 - Formulário de uma busca por CDs. 152

Figura 36 - Resposta do sistema a uma busca por CDs. 153

Figura 37 - Detalhes de um CD. 153

Figura 38 - Formulário de uma busca de produtos. 154

Figura 39 - Resposta do sistema a uma busca por produtos. $\quad 155$

Figura 40 - Árvores de classificação de conceitos. 158

Figura 41 - Diagramas de relações binárias. 159

Figura 42 - Classes responsáveis pela interface principal do sistema. 173

Figura 43 - Classes responsáveis pela interface da opção de listar as triplas da máquina de inferência.

Figura 44 - Classes responsáveis pela interface de construção de formulários.

Figura 45 - Classes responsáveis pela interface da listagem dos produtos.

Figura 46 - Classes responsáveis pela interface da apresentação dos detalhes dos produtos.

Figura 47 - Classe responsável pela interface com a base de conhecimento. 
Figura 48 - Classes responsáveis por controlar as classes das ontologias.

Figura 49 - Classes responsáveis por formular as perguntas de busca por produto.

Figura 50 - Classes responsáveis por formular a pergunta para indicação de produto a um perfil de consumidor.

Figura 51 - Classes responsáveis por formular a pergunta para obtenção de todas as triplas da máquina de inferência.

Figura 52 - Classes responsáveis pela fábrica de uma família de produtos.

Figura 53 - Formulário Busca CD. 188

Figura 54 - Lista Busca CD. 189

Figura 55 - Buscar Atributos Exclusivos Tipo Produto CD. 190

Figura 56 - Buscar Atributos Comuns Todos Produtos - parte 1

Figura 57 - Buscar Atributos Comuns Todos Produtos - parte 2

Figura 58 - Buscar Atributos Comuns Todos Produtos - parte $3 \quad 193$

Figura 59 - Obter Descrição Produtos CD - parte 1.

Figura 60 - Obter Descrição Produtos CD - parte 2. 


\section{Lista de Tabelas}

Tabela 1 - Classes RDF/RDF Schema predefinidas na especificação (Brickley \& Guha, 2002).

Tabela 2 - Propriedades RDF/RDF Schema predefinidas na especificação (Brickley \& Guha, 2002).

100

Tabela 3 - Tags utilizadas para a declaração de ontologias em SHOE. 114 Tabela 4 - Tags utilizadas para a declaração de instâncias em SHOE. 114 Tabela 5 - Comparação das funcionalidades para descrição de conceitos.

Tabela 6 - Comparação das funcionalidades para descrição de relacionamentos e funções.

Tabela 7 - Comparação das funcionalidades para descrição de axiomas.

Tabela 8 - Comparação das funcionalidades para descrição de instâncias.

Tabela 9 - Documento sobre o objetivo da ontologia com breve descrição do domínio.

Tabela 10 - Dicionário de conceitos - Taxinomia Produto.

Tabela 11 - Dicionário de conceitos - Taxinomia Gênero Produto. 160

Tabela 12 - Dicionário de conceitos - Taxinomia Pessoa. 161

Tabela 13 - Dicionário de conceitos - Taxinomia Loja. 162

Tabela 14 - Dicionário de conceitos - Taxinomia Local. 162

Tabela 15 - Dicionário de conceitos - Taxinomia Unidade Monetária. 162

Tabela 16 - Dicionário de conceitos - Taxinomia Forma Pagamento. 162

Tabela 17 - Tabela de relações binárias - Taxinomia Produto. 163

Tabela 18 - Tabela de relações binárias - Taxinomia Gênero Produto. 163

Tabela 19 - Tabela de relações binárias - Taxinomia Loja. 163

Tabela 20 - Tabela de atributos de instância - Conceito Produto. 164

Tabela 21 - Tabela de atributos de instância - Conceito Livro. 164

Tabela 22 - Tabela de atributos de instância - Conceito CD. 165 
Tabela 23 - Tabela de atributos de instância - Conceito Local.

Tabela 24 - Tabela de atributos de instância - Conceito Loja.

Tabela 25 - Tabela de atributos de instância - Conceito GeneroProduto.

Tabela 26 - Tabela de atributos de instância - Conceito Pessoa.

165

Tabela 27 - Tabela de atributos de instância - Conceito

UnidadeMonetaria.

Tabela 28 - Tabela de atributos de instância - Conceito

FormaPagamento.

Tabela 29 - Tabela de axiomas - Conceito Pessoa.

168

Tabela 30 - Tabela de axiomas - Conceito Produto.

168

Tabela 31 - Tabela de instâncias - Conceito GeneroLiterario. 169

Tabela 32 - Tabela de instâncias - Conceito GeneroMusical. 169

Tabela 33 - Tabela de instâncias - Conceito UnidadeMonetaria. 170

Tabela 34 - Tabela de instâncias - Conceito FormaPagamento.

170 


\section{Lista de Códigos}

Código 1 - Características principais de um documento XML.

Código 2 - Partes de um documento XML. Documento possui somente prólogo e corpo.

Código 3 - Exemplo de utilização de namespaces.

Código 4 - Prólogo e elemento raiz de um documento RDF típico. $\quad 89$

Código 5 - Documento RDF da figura 19.

Código 6 - Versão serializada e versão abreviada de um código. $\quad 91$

Código 7 - Versão serializada e versão abreviada de um código. $\quad 91$

Código 8 - Versão serializada e versão abreviada de um código. $\quad 92$

Código 9 - Documento RDF que instância uma Bag de literais. 95

Código 10 - Documento RDF que instância uma Bag de recursos. 95

Código 11 - Documento RDF Schema que especifica a ontologia se seres.

Código 12 - Documento RDF que instância conceitos do esquema do código 11.

Código 13 - Cabeçalho de um documento DAML+OIL.

Código 14 - Elementos de classe.

Código 15 - Restrições de propriedade.

Código 16 - Elementos de propriedade.

Código 17 - Documento HTML que descreve um livro da Livraria Dirigida.

Código 18 - Documento RDF que descreve o livro da Livraria Dirigida. 130 Código 19 - Definição da propriedade ehRelacionaodo na ontologia de produto.

Código 20 - Documento RDF que descreve o livro da Livraria Dirigida. 134 